\title{
A comparative study of chromosome G-banding using trypsin, papain, and pretreatment with emulphogene
}

\author{
GHIZALA KHALID, HAVA NEUMANN 1 , R. J. FLEMANS, AND \\ F. G. J. HAYHOE \\ From the Department of Haematological Medicine, Cambridge University, UK
}

SUMMARY G-banding of chromosome metaphase preparations derived from haemic cells of healthy individuals and from patients with acute myeloid leukaemia was performed with the aid of trypsin, papain, and pretreatment of the chromosome spreads with emulphogene before proteolytic digestion. Papain digestion revealed more distinguishable bands than did trypsin digestion. Pretreatment of the chromosome spreads with emulphogene greatly enhanced the number of distinguishable bands for both enzymes. The combination of pretreatment with emulphogene and digestion with papain revealed optimal numbers of bands for individual chromosomes essentially identical with those agreed at the Paris Conference 1971. The use of the emulphogene-papain technique appears also to offer an advantage in the banding of chromosomes from leukaemic cells.

The existence of specific banding patterns which enable individual human chromosomes to be identified was first demonstrated by Caspersson et al. (1968) using quinacrine fluorescence. Similar banding could also be shown by Giemsa staining of chromosome preparations previously exposed to proteolytic digestion using pronase (Dutrillaux et al., 1971) or trypsin (Seabright, 1971). The nature of the banded material is uncertain, although it seems possible that the fluorescent bands may represent condensed DNA segments rich in adenine-thymine base pairs, to which quinacrine binds preferentially (Pachmann and Rigler, 1972), while the equivalent dark bands in trypsin-Giemsa preparations may become visible because the condensed chromatin has associated proteins different in kind and in susceptibility to proteolytic digestion from those linked to light band segments of uncondensed chromatin.

Trypsin-Giemsa banding has enormously increased the discriminatory power of cytogenetic techniques and has been used widely in a great range of disease in man (Lawler and Reeves, 1976) including many neoplastic states. Increasingly, evi-

${ }^{1}$ On sabbatical leave from the Weizman Institute of Science, Rehovot, Israel

Received for publication 21 November 1978 dence has emerged of the existence of non-random chromosomal abnormalities in certain of these states, including the leukaemias (Rowley, 1978). In this field, however, difficulties have arisen in obtaining good banded preparations because the chromosomes of leukaemic cells often show poor morphological outlines with a woolly or fuzzy appearance so that the bands do not stain well and are not clearly separable. This appearance may reflect an unusual concentration of proteins around leukaemic chromosomal DNA, less susceptible to proteolysis in the G-banding procedure than the DNA-associated protein in normal spreads.

In the course of a general study of technical methods which might improve the quality of banding, especially in acute leukaemic chromosomes, we have explored the use of papain as an alternative to trypsin in the Giemsa banding procedure and the possible advantages of preliminary treatment of chromosome preparations with the surface active agent, emulphogene. The reasons for this approach were, briefly, as follows. Proteins attached to the negatively charged DNA are mainly histones and basic proteins rich in lysine and arginine. Trypsin catalyses the hydrolysis of peptide bonds whose carbonyl group is donated by either lysine or arginine. Cleavage at one specific amino acid occurs with trypsin, when the $\epsilon$-amino groups of the protein to be digested are blocked, and tryptic 
digestion takes place only at the arginine residues. This technique would be difficult to apply in a heterogeneous system. Katchalski et al. (1961), however, showed that papain, at high $\mathrm{pH}$ values, in the range $\mathrm{pH} 8.0$ to $12 \cdot 0$, catalyses hydrolysis at lysine residues only. This restricted action of papain may readily be applied in heterogeneous systems and might permit a more controlled and selective digestion of histones and other basic proteins around the chromosomal DNA.

Pretreatment of chromosome spreads with emulphogene might be expected to loosen the noncovalently complexed proteins from DNA by weakening the strong attachment of proteins to it, so allowing the subsequent enzymatic hydrolysis of peptide links to lead to more effective stripping of large oligopeptides from the DNA, with consequent improvement in banding patterns on Leishman or Giemsa staining.

\section{Material and methods}

A serial chromosome G-banding study was made on blood and bone marrow from normal subjects and from patients with acute or chronic myeloid leukaemias using pretreatment with (1) trypsin (lot 0153-59, purchased from Difco Laboratories, Detroit, Michigan, USA), (2) emulphogene (the non-ionic detergent, alkoxypoly(ethylenoxy)ethanol, BC720, purchased from General Aniline and Film Corp, New York, USA) and trypsin, (3) papain ( 2 crystallised, lot 3125 from Sigma Chemical Co), and (4) emulphogene and papain.

Short-term cultures of whole blood and bone marrow were used for making chromosome preparations. $0.1 \mathrm{ml}$ of $0.04 \mathrm{mg} / \mathrm{ml}$ of colchicine was added 2 hours before harvest to arrest metaphases. A $0.075 \mathrm{M} \mathrm{KCl}$ solution for 10 minutes was used as the hypotonic medium. The fixative ( 3 parts methanol: 1 part glacial acetic acid) was changed three times. Cells suspended in fixative were dropped onto clean dry slides by means of a pipette, gently blown on the top, and allowed to air dry. A series of slides was made from each cell suspension for subjection to the four procedures described. Chromosomes were G-banded using a modification of Seabright's (1971) technique.

In the case of papain, the chosen preparation was flooded with $5 \mathrm{ml}$ of $0.1 \mathrm{M}$ sodium phosphate buffer, pH 8.0 , containing $0.5 \mu \mathrm{g}$ papain and $0.9 \mu \mathrm{g}$ sodium phosphorothioate. The latter compound was used for activation of papain (Neumann et al., 1967). The slides were exposed to this solution at room temperature for 60 seconds in the case of normal preparations and for 30-45 seconds for leukaemic cell preparations. They were rinsed thoroughly with isotonic saline and stained for 5-7 minutes with Leishman (BDH) diluted 1/4 with $\mathrm{pH} 6.8$ buffer. The slides were then rinsed with buffer, blotted dry, and observed under the microscope.

Trypsin and papain digestion were also performed on the same preparations after pretreatment with emulphogene solution. The slide to be banded was dipped in $0.1 \% \mathrm{w} / \mathrm{v}$ of emulphogene water solution for 2 minutes before trypsin or papain digestion.

\section{Results}

The results for each preparative method were assessed by comparing the banding patterns achieved with the composite diagrams of 'optimal' banding approved at the Paris Conference (1971). In each case at least 20 banded metaphases with good chromosome separation were studied, and typical examples of the best banding patterns found following the different preparative procedures were photographed and karyotyped (Figs 1-4). The results are summarised in the Table.

In the chromosome preparations made by the standard trypsin treatment (Fig. 1, Table) we obtained the optimal number of demonstrable

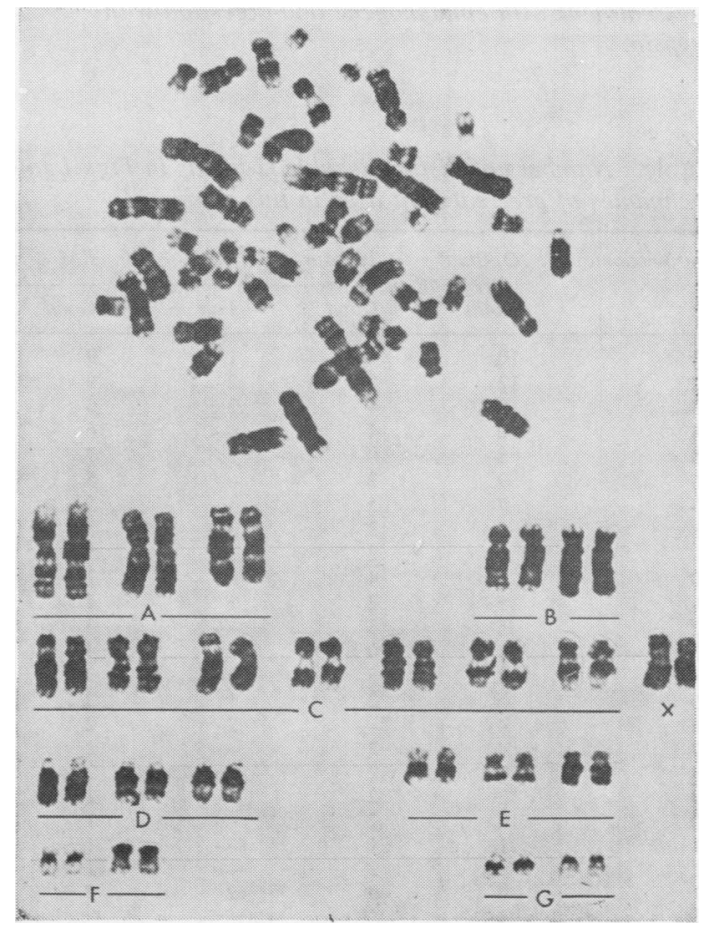

Fig. 1 Normal metaphase spread, G-banded after trypsin digestion. 


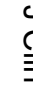

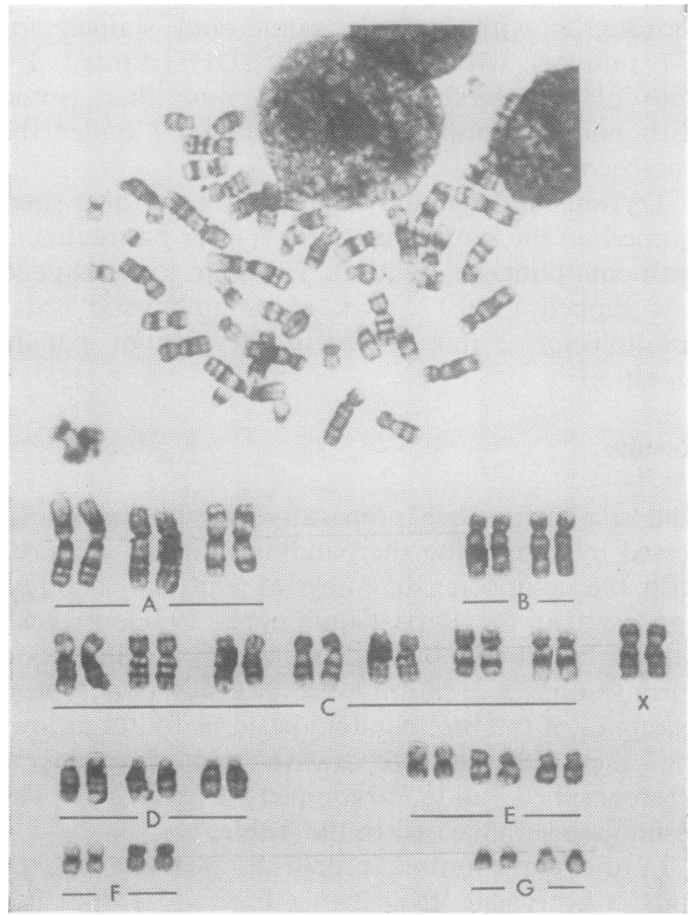

Fig. 2 Normal metaphase spread, G-banded after pretreatment with emulphogene and digestion with trypsin.

Table Numbers of distinguishable G-bands in Figs 1 to 4, representative of the patterns found with the four techniques of pretreatment used in this study

\begin{tabular}{|c|c|c|c|c|c|c|c|c|c|}
\hline \multirow[t]{2}{*}{ Chromosome } & \multirow{2}{*}{$\begin{array}{l}\text { Optimal } \\
\text { number of } \\
\text { bands* }\end{array}$} & \multicolumn{2}{|c|}{ Trypsin } & \multicolumn{2}{|c|}{$E M+t r y p s i n$} & \multicolumn{2}{|c|}{ Papain } & \multicolumn{2}{|c|}{$E M+$ papain } \\
\hline & & Good & Fair & Good & Fair & Good & Fair & Good & Fair \\
\hline 1 & 10 & 6 & 1 & 9 & - & 9 & 2 & 10 & - \\
\hline 2 & 12 & 5 & 2 & 9 & - & 7 & 3 & 12 & - \\
\hline 3 & 10 & 4 & 2 & 6 & 1 & 7 & 2 & 10 & - \\
\hline 4 & 10 & 4 & - & 6 & - & 8 & 一 & 9 & 1 \\
\hline 5 & 8 & - & 5 & 7 & 1 & 7 & - & 8 & - \\
\hline 6 & 9 & 2 & - & 5 & - & 7 & - & 9 & - \\
\hline 7 & 7 & 5 & - & 5 & 1 & 6 & - & 7 & 一 \\
\hline 8 & 5 & 2 & 3 & 3 & 2 & 4 & 3 & 7 & - \\
\hline 9 & 6 & 2 & 1 & 5 & - & 5 & - & 6 & - \\
\hline 10 & 5 & 4 & - & 7 & - & 6 & - & 5 & - \\
\hline 11 & 5 & 2 & 1 & 4 & - & 5 & - & 5 & - \\
\hline 12 & 5 & 3 & - & 3 & $i$ & 5 & - & 5 & - \\
\hline 13 & 4 & 0 & 2 & 4 & 1 & 5 & 1 & 5 & - \\
\hline 14 & 4 & 3 & 1 & 3 & 1 & - & 2 & 5 & - \\
\hline 15 & 5 & 2 & 1 & 3 & & 3 & 2 & 4 & 1 \\
\hline 16 & 4 & - & 2 & 2 & - & 3 & 1 & 4 & \\
\hline 17 & 4 & 2 & & 2 & 1 & 4 & & 4 & - \\
\hline 18 & 2 & 2 & - & 2 & 1 & 4 & - & 4 & - \\
\hline 19 & 2 & 2 & - & 1 & - & 3 & - & 2 & 1 \\
\hline 20 & 2 & 2 & - & 1 & 1 & 1 & - & 2 & $i$ \\
\hline 21 & 1 & 1 & & 1 & - & 2 & 一 & 1 & 1 \\
\hline 22 & 1 & 2 & - & 1 & 1 & 2 & - & 2 & \\
\hline $\mathbf{X}$ & 6 & 3 & 2 & 5 & 1 & 5 & 1 & 6 & - \\
\hline $\mathbf{Y}$ & 1 & & & & & & & & \\
\hline
\end{tabular}

*Column 2 shows the optimal number of bands agreed at the Paris Conference, 1971.

Fig. 3 Normal metaphase spread, G-banded after papain digestion.

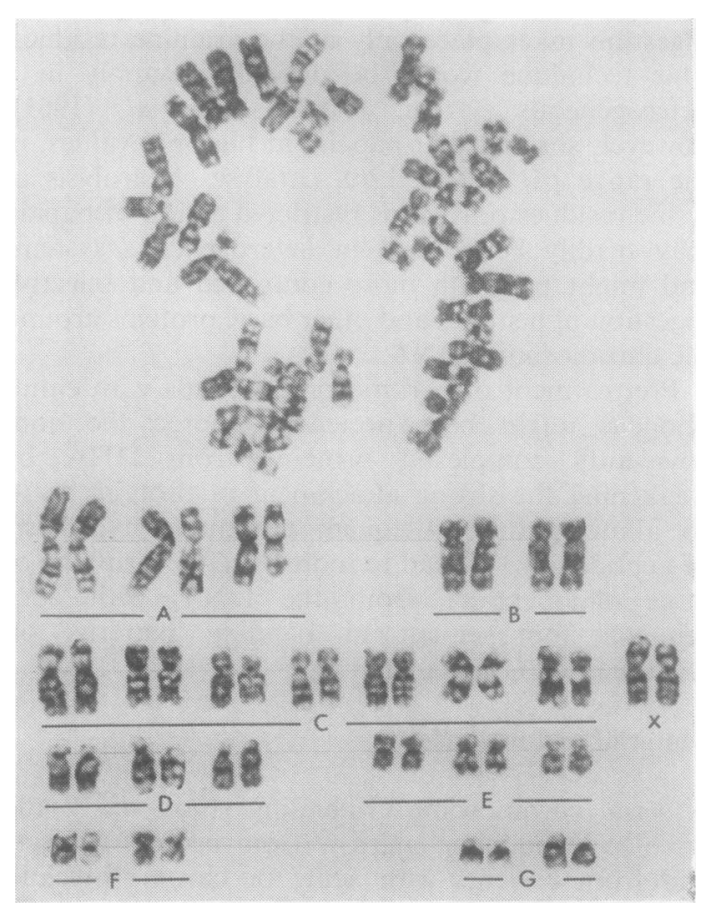




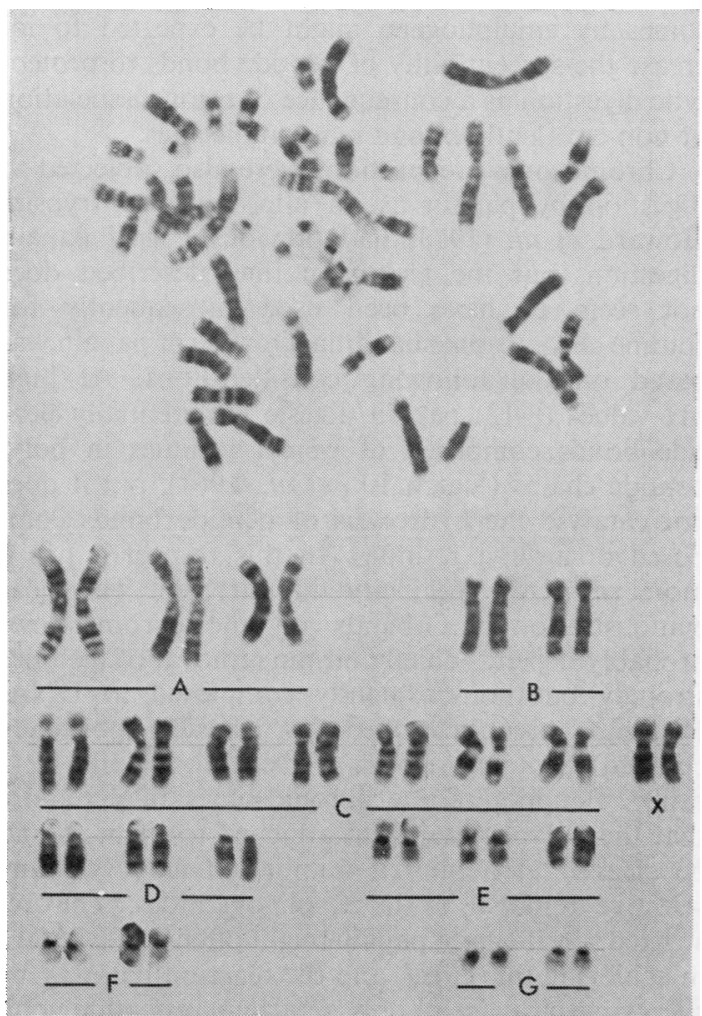

Fig. 4 Normal metaphase spread, G-banded after pretreatment with emulphogene and digestion with papain.

bands only for chromosomes $8,14,18,19$, and 22 . Pretreatment with emulphogene, followed by trypsin digest, resulted in an improved resolution of most of the chromosome bands. The number of distinguishable bands was found to be increased (see Fig. 2, Table) and for certain chromosomes came very close to the optimal number. However, the optimal number of bands was achieved only for chromosomes $5,8,14,18,19,20,22$, and $X$. In the case of papain digestion, the number of bands for most of the chromosomes was found to be greater than with tryptic digestion. The optimal number of bands was obtained for chromosomes $1,5,10,11,12,13,15,16$, 17, 19, 20, 21, 22, and X (Fig. 3, Table). Pretreatment of the slides with emulphogene, followed by papain digestion, resulted in nearly the exact optimal number of bands required for characterisation of each chromosome (Fig. 4, Table).

In some chromosomes more bands than the optimal number could be distinguished. At this stage of our research we cannot be certain of the extent of reproducibility of these additional bands.
However, confirmation of the above findings might establish these bands as a useful marker for the chromosomes concerned (see Table).

Because of problems of fuzziness and poor banding of chromosomes derived from patients with leukaemias, we extended the comparative study to preparations from a patient with acute myeloid leukaemia. The results are illustrated in Figure 5. Pretreatment with emulphogene, for both tryptic and papain digestion, greatly improved the number of distinguishable bands. The emulphogenepapain treatment gave better resolution for most of the chromosomes.

These results suggest that pretreatment with emulphogene may greatly facilitate the demonstration of chromosome bands.

\section{Discussion}

Since the chromosome spreads used in this comparative study were all prepared at the same time from the same cell suspensions and subjected to pretreatment after the same inte:vals, the differences between the banding patterns obtained are reasonably attributable to the action of emulphogene and

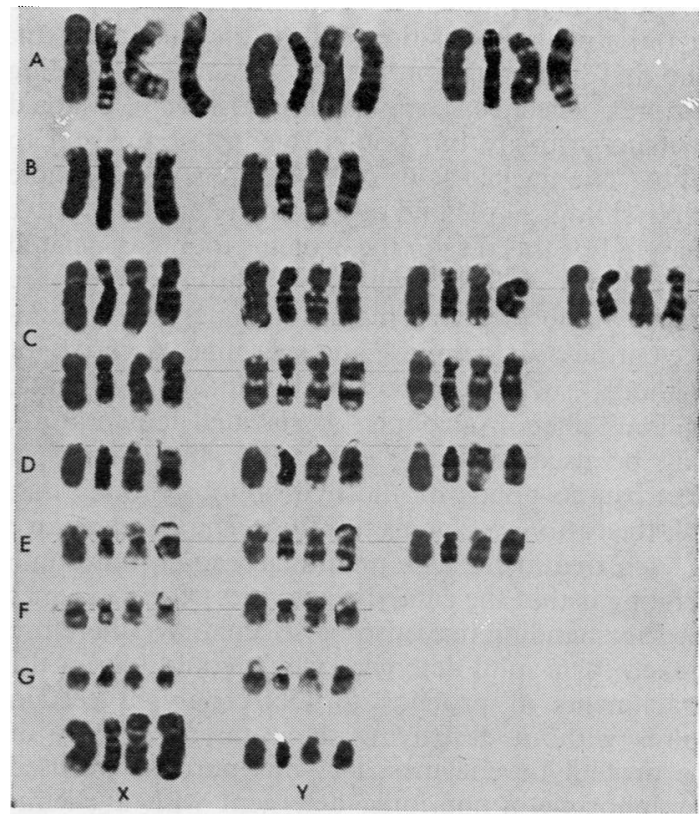

Fig. 5 Karyotypes from chromosome preparations of acute myeloid leukaemia cells showing $G$-banded appearances after treatment with trypsin (column 1), emulphogene and trypsin (column 2), papain (column 3), and emulphogene and papain (column 4). 
the differing proteolytic activities of papain and trypsin.

The optimal conditions for digestion of proteins and polypeptides in solution by proteolytic enzymes are well established. The procedure in most cases involves the opening of disulphide bonds either by reduction or by oxidation and transformation of the native conformation of the protein, containing a high percentage of helical structure, to random coil conformation. This transformation can be achieved either by acid or organic solvent treatment, or by exposure of the protein solution to elevated temperatures. It is also customary to remove the noncovalently bound ligands by ionic or non-ionic detergents. All the above manipulations can be performed under well controlled conditions. Thus for protein in solution one is able to obtain for each protein quantitative information about the points of cleavage in the polypeptide chains and the composition of each individual peptide fragment. In contrast, the digestion of cellular, membrane or chromosome bound proteins involves a process of trial and error. There is no way to determine in advance the optimal conditions for proteolytic digestion for any one particular cell or chromosome. Consequently, these fields of study are open to the introduction of new techniques.

The proteins attached to chromosomal DNA are of unknown composition and spatial arrangement. The first 'manipulation' of chromosome G-banding requires selective removal of positively charged proteins strongly but non-covalently complexed to DNA. The nucleic acids of DNA are strongly acidic at physiological $\mathrm{pH}$ and carry a high density negative charge. For this reason the proteins found associated with DNA in the cells and chromosomes are mostly basic proteins, mainly histones, or histonelike entities, oligoamines, and spermine. A variety of methods, such as exposure to dodecyl sulphate, aqueous phenol, alcohols, or elevated temperature, may be used to break either the strong multiple electrostatic bonds or the hydrophobic bonds that link the cationic proteins to DNA. The disadvantage of such treatments as a preliminary to chromosome banding is that the catalytic activities of the enzymes used for banding may also be affected. We therefore looked for a mild detergent which could loosen the attachments of proteins to DNA only at certain points without destroying the catalytic activity of the proteolytic enzymes. For this purpose we used emulphogene, a non-ionic detergent with a chemical composition: alkoxy (ethylenoxy) ethanol. This detergent was first used for extraction of rhodopsin from rod outer segments isolated from retina, without destroying its biological properties (O'Brien et al., 1972). Similarly, pretreatment of the chromo- somes by emulphogene might be expected to increase the susceptibility of peptide bonds to proteolytic digestion as a consequence of prior dissociation of non-covalently bound macromolecules.

Chromosome preparations were also subjected to digestion by papain as an alternative to trypsin. Howard et al. (1973) had previously used papain digestion, but the technique they described does not seem to have been used subsequently for routine chromosome banding. Our use of papain was based on the following considerations. At high $\mathrm{pH}$ values (8-12) papain digests preferentially peptide bonds composed of lysinyl residues in polypeptide chains (Katchalski et al., 1961), but it does not catalyse the hydrolysis of peptide bonds composed of arginyl residues. In this respect it has a more restricted specificity than trypsin. The clear demonstration of G-bands on the chromosomes probably depends chiefly on the removal of histones strongly but non-covalently complexed to DNA. Histones are heterogeneous, basic (positively charged) proteins rich in lysine and arginine (2:1 ratio). Banding of the chromosomes may require that the polypeptide chain attached to them should be cleaved at a limited number of sites, yielding selective removal of large oligopeptides. The restricted specificity of papain might offer an advantage in achieving this effect, and the reaction pathway by successive cleavage of only a particular peptide bond might explain the clearer banding patterns, specific for each chromosome, achieved by this preparative method.

Whatever the mechanism, a clear improvement in resolution of bands followed the use of emulphogene as a preliminary to proteolytic digestion with either trypsin or papain, and the general superiority of papain over trypsin as a digestive agent for G-banding was manifest in both normal karyotypes and those from leukaemic cells.

We are grateful to Miss J. Thompson for typing the manuscript. Ghizala Khalid holds a scholarship from the Royal Commission for the Exhibition of 1851.

\section{References}

Caspersson, T., Farber, S., Foley, G. E., Kudynowski, J., Modest, E. J., Simonsson, E., Wagh, U., and Zech, L. (1968). Chemical differentiation along metaphase chromosomes. Experimental Cell Research, 49, 219-222.

Dutrillaux, B., de Grouchy, J., Finaz, C., and Lejeune, J. (1971). Mise en évidence de la structure fine des chromosomes humains par digestion enzymatique (pronase en particulier). Comptes rendus hebdoma- 
daires des Séances de l'Académie des Sciences, D, 273, 587-588.

Howard, P. N., Stoddard, G. R., and Seely, J. R. (1973). Banding of human chromosomes treated with papain. Clinical Genetics, 4, 162-165.

Katchalski, E., Levin, Y., Neumann, H., Riesel, E., and Sharin, N. (1961). Studies of the enzymatic hydrolysis of poly-a-amino acids. Bulletin of the Research Council of Israel, Section A, Chemistry, 10A, 159-171.

Lawler, S. D., and Reeves, B. R. (1976). Chromosome studies in man: past achievements and recent advances. Journal of Clinical Pathology, 29, 569-582.

Neumann, H., Shinitzky, M., and Smith, R. A. (1967). The activation of papain and ficin by phosphorothioate. Biochemistry, 6, 1421-1428.

O'Brien, P. J., Muellenberg, C. G., and Bungenberg de Jong, J. J. (1972). Incorporation of leucine into rhodopsin in isolated bovine retina. Biochemistry, 11, 64-70.

Pachmann, U., and Rigler, R. (1972). Quantum yield of acridines interacting with DNA of defined base sequence. Experimental Cell Research, 72, 602-608.

Paris Conference (1971). Standardization in human cytogenetics. Birth Defects: Original Article Series. Vol. 8, no. 7. National Foundation, New York.

Rowley, J. D. (1978). The cytogenetics of acute leukaemia. Clinics in Haematology, 7, 385-406.

Seabright, M. (1971). A rapid banding technique for human chromosomes (Letter). Lancet, 2, 971-972.

Requests for reprints to: Professor F. G. J. Hayhoe, Department of Haematological Medicine, University of Cambridge Clinical School, Hills Road, Cambridge CB2 2QL, UK. 